\title{
Multimorbilidad en los servicios médicos hospitalarios: un problema clínico y de gestión
}

\section{Multimorbidity in hospital medical services: a clinical and management problem}

\author{
María Matesanz Fernández¹, Iria Î́niguez Vázquez¹, David Rubal Bran¹, Sonia Pértega Díaz², \\ Jesús Conde Freire ${ }^{3}$, Emilio Casariego Vales ${ }^{1}$ \\ ${ }^{1}$ Servicio de Medicina Interna. Hospital Universitario Lucus Augusti. SERGAS. Lugo \\ ¿Unidad de epidemiología clínica y bioestadística. Complexo Hospitalario Universitario de A Coruña (CHUAC). SERGAS. A Coruña \\ ${ }^{3}$ Servicio de Documentación Médica. Hospital Universitario Lucus Augusti SERGAS. Lugo
}

\section{Resumen}

INTRODUCCIÓN: La multimorbilidad, a pesar de ser muy frecuente y tener mucha repercusión socio-sanitaria, es un fenómeno poco estudiado.

PACIENTES Y METODOS: Estudio de la totalidad de ingresos hospitalarios en los servicios del área médica de adultos en un Hospital General entre 2000 y 2009. Los datos se obtuvieron de la base de datos general del centro. Se crearon nuevas variables con la integración de otras bases del servicio y tras el análisis y compactación de los datos existentes. En el análisis estadístico se utilizaron técnicas habituales de la estadística descriptiva.

RESULTADOS: En los 10 años en estudio se registraron 111.123 ingresos correspondientes a 52269 pacientes (varones 55,9\%, edad media 68,6 -DT 17,2- , mortalidad acumulada 26,5\%) distribuidos en 12 servicios hospitalarios. El $51,2 \%$ de los pacientes sufrían al menos una de las siguientes patologías: Cardiopatía isquémica (12,4 \%), insuficiencia cardiaca (15,2\%), Demencia (9,8 \%), cáncer (18,1\%) y EPOC (16,4 \%). En conjunto, sufrían dos o más de estas patologías 8597 pacientes, sumando 30598 ingresos, 2818 fallecimientos hospitalarios (32,8\%) y una estancia media de 12,4(DT $7,1)$ días. Por asociaciones de patologías la más frecuente fue EPOC/ insuficiencia cardiaca (1355 pacientes, 5330 ingresos) y la menos habitual la asociación de todas ellas (18 pacientes, 125 ingresos).

CONCLUSIÓN: La multimorbilidad supone un desafío clínico y un importante problema de gestión. Nuestro estudio identifica subgrupos de pacientes que suponen un porcentaje importante de la actividad hospitalaria y donde es posible establecer programas que mejoren su calidad de vida reduciendo sus ingresos hospitalarios.

PALABRAS CLAVE: Multimorbilidad, mortalidad, calidad asistencial

\section{Introducción}

Se define multimorbilidad (MM) como la concurrencia de dos o más patologías crónicas en un paciente ${ }^{1}$. Entre los pacientes ingresados en un hospital esta situación es muy frecuente y se estima que la media de problemas médicos por paciente es de 5 y que solo un 15\% tiene un único problema médico bien definido. Sin embargo, entre los hospitalizados mayores de 65 años, el promedio es de 6,4 enfermedades crónicas por paciente ${ }^{2}$. En España, un estudio realizado sobre un millón de altas hospitalarias apreció que los pacientes ingresados en Medicina Interna tenían un promedio de 5,84 patologías médicas ${ }^{3}$. Además de la edad, esta situación se incrementa cuando existen determinadas patologías. Así, se apreció que hasta el 49\% de los pacientes con Insuficiencia cardíaca tenía n 3 o más patologías concomitantes ${ }^{4}$.

\section{Abstract}

INTRODUCTION: Multimorbidity, a very frequent and with a big sociosanitary repercussion, is a little studied situation.

PATIENTS AND METHODS: A study of totality of adults hospital discharges in medical services between 2000 and 2009 was conducted. The study variables were obtained from hospital records. New variables were created with integration of other medical records and after derivation from the original variables. A descriptive statistical analysis was conducted.

RESULTS: In 10 years-study and 12 adults hospital medical services, 111.123 discharges in 52269 patients (male 55,9\%, mean age 68,6 SD 17,2- , accumulated mortality $26,5 \%$ ) were registered. $51,2 \%$ of patients had at least one of following: coronary ischemic disease $(12,4 \%)$, heart failure $(15,2 \%)$, dementia $(9,8 \%)$, cancer $(18,1 \%)$ y COPD $(16,4$ \%). Totally, 8597 had at least two of them, generating 30598 discharges, 2818 in-hospital deaths and mean length stay 12,4 (SD 7,1) days. The most frequent association was COPD/heart failure (1355 patients, 5330 admissions) and the least all together association (18 patients, $125 \mathrm{ad}-$ missions).

CONCLUSSION: Multimorbidity is a clinical challenge and a management problem. Our study identifies groups of patients with a great repercussion over hospital activity. It's possible to establish programs to improve quality life and reducing their in-hospital admissions.

KEY WORDS: Multimorbidity, mortality, quality of health care

En contraste con su enorme frecuencia y repercusión sobre los servicios sanitarios, es un fenómeno poco estudiado. Si bien es claro que los pacientes con MM tienen una mayor mortalidad, mayor discapacidad y peor calidad de vida así como un incremento de uso de servicios sanitarios y sociales ${ }^{5}$, las limitaciones apreciadas en la mayoría de los estudios son, todavía, notables. A nivel clínico, las diferentes combinaciones de MM, su proceso de asociación y desarrollo en el tiempo así como sus posibles interacciones y sinergias son, hoy en día, básicamente desconocidos. Como consecuencia, de muchos aspectos de su manejo en la asistencia diaria son claramente mejorables ${ }^{5,6}$ y el peso asistencial que supone es desconocido. Por ello, el objetivo de este estudio fue conocer, a través de las asociaciones de 5 patologías más frecuentes, el peso de la MM en los servicios Médicos de un Hospital General. 


\section{Pacientes y métodos}

Estudio de la totalidad de ingresos hospitalarios efectuados en los Servicios del área médica del Hospital Xeral-Calde de Lugo entre Enero de 2000 hasta Diciembre de 2009 inclusive. Este Centro disponía, en la época en estudio, de 769 camas repartidas entre 3 Centros Hospitalarios, cubriendo la asistencia de una población de unos 240.000 habitantes. Este Complexo Hospitalario se cerró en diciembre de 2010 al trasladarse a un nuevo Centro Hospitalario. El área médica estaba formada por los siguientes doce servicios: Cardiología, Endocrinología, Reumatología, Oncología, Neumología, Digestivo, Neurología, Nefrología, Geriatría, Unidad de Media Estancia, Infecciosas y Medicina Interna. Los datos se obtuvieron de la base CMBD del centro. El elemento básico en esta primera parte de estudio fue el ingreso hospitalario y contenía las siguientes variables: número de historia clínica, servicio de ingreso, ayuntamiento de procedencia, sexo, fecha de nacimiento, edad al ingreso, fecha de ingreso, fecha de alta, día de alta, estancia (en días), destino al alta (incluyendo fallecimiento), GRD, diagnóstico principal, diagnóstico secundarios.

Tabla 1. Relación de las 25 patologías crónicas más frecuentes del Área Médica

\begin{tabular}{|l|c|}
\hline Enfermedad & Frecuencia \\
\hline Hipertensión Arterial & $40,8 \%$ \\
\hline Diabetes Mellitus & $19,5 \%$ \\
\hline Dislipemia & $18,7 \%$ \\
\hline Cáncer de cualquier localización & $18,1 \%$ \\
\hline Fibrilación auricular & $17,8 \%$ \\
\hline Enfermedad Obstructiva Crónica & $16,4 \%$ \\
\hline Insuficiencia Cardíaca & $15,2 \%$ \\
\hline Ictus & $13,2 \%$ \\
\hline Cardiopatía Isquémica & $12,4 \%$ \\
\hline Trastornos del Humor & $9,9 \%$ \\
\hline Demencia & $9,8 \%$ \\
\hline Obesidad & $7 \%$ \\
\hline Alcoholismo & $6,2 \%$ \\
\hline Enfermedad Renal crónica & $6,1 \%$ \\
\hline Otras enfermedades Neumológicas & $6 \%$ \\
\hline Valvulopatía Aórtica & $5,2 \%$ \\
\hline Problemática Social & $4,9 \%$ \\
\hline Valvulopatía Mitral & $4,5 \%$ \\
\hline Epilepsia & $4,2 \%$ \\
\hline Asma & $3 \%, 8 \%$ \\
\hline Parkinson & $3,5 \%$ \\
\hline Ansiedad & $2,6 \%$ \\
\hline Miiocardiopatía & $2,5 \%$ \\
\hline Hepatopatía no Alcohólica & \\
\hline Hepatopatía Alcohólica & $2 \%$ \\
\hline
\end{tabular}

Cuatro facultativos revisaron la base buscando errores (datos incompletos, errores de clasificación, errores de tecleo...). Para ello se verificaron todos los casos de manera individual, consultando la historia clínica cuando fue preciso, hasta que se consideró que la base de datos estaba libre de errores. Posteriormente cada uno de los diagnósticos integrados en cada uno de los ingresos se clasificó como agudo o crónico, utilizando como criterio el acuerdo entre todos los facultativos participantes en el estudio.

Seguidamente se clasificaron los distintos episodios de ingreso según las clasificaciones previamente utilizadas en este tipo de estudios, para ello se utilizaron los criterios de Charlson et al y Cumullative IIIness Rating Scale ${ }^{8}$. Para establecer las patologías crónicas más prevalentes se utilizaron Ios criterios de The German MultiCare-study 9 .

En un segundo tiempo se reconvirtió la base de datos de episodios de hospitalización en una base de pacientes. Para ello se constituyó una matriz donde cada fila incluía a un único paciente, con sus sucesivos ingresos, siendo cada columna una variable distinta. Ello permitió calcular otras variables secundarias (por ejemplo, tiempo entre ingresos)

Posteriormente se añadieron datos que provenían de otras bases de datos del centro (visitas a Consultas Externas, último contacto con el hospital, ...). Para finalizar se realizó un control de errores en la búsqueda de inconsistencia o de datos imposibles y se corrigieron de forma sucesiva hasta conseguir la depuración completa de todos los datos.

Puesto que el objeto del estudio era conocer el peso de la MM sobre el trabajo asistencial del área médica, se seleccionaron 5 patologías de entre las de mayor relevancia clínica. La selección se realizó por consenso del grupo investigador de entre las 25 de mayor prevalencia. Estas patologías fueron: Insuficiencia cardíaca (ICC), Cardiopatía isquémica (CI), Enfermedad pulmonar obstructiva crónica (EPOC), Demencia y Cáncer de cualquier localización. En el análisis estadístico se utilizaron técnicas habituales de estadística descriptiva. En el manejo de los datos se utilizó el paquete estadístico SPSS.

\section{Resultados}

Entre el 1 de enero de 2000 y el 31 de diciembre de 2009 se han producido un total de 111.123 ingresos en los doce servicios que componen el área médica del Hospital. Estos ingresos correspondieron a un total de 52.269 pacientes. De ellos, $29.521(56,5 \%)$ ingresaron una única vez y el resto (22.748 pacientes) 20 más veces. En la serie general el 55,9\% eran varones, la edad media 68,6 (DT 17,3) años y la mortalidad acumulada en los sucesivos ingresos fue del 16,5\%.

Se determinaron las 25 patologías crónicas más frecuentes (Tabla 1). Los pacientes que sufrieron al menos una de las 5 patologías seleccionadas ( ICC, Cl, EPOC , Demencia y Cán- 
cer) fueron 26.781 , esto es el $51,2 \%$ del total de pacientes ingresados en los 10 años en estudio. De entre ellos, 18.173 $(67,8 \%)$ presentaban una única de estas 5 enfermedades y $8.577(32,0 \%)$ pacientes presentaban diferentes combinaciones de al menos dos de estas patologías. Por el contrario, solo 25.488 (48.8\%) pacientes no presentaban ninguna de estas patologías. Este grupo de pacientes tenían una edad media de 61,9 años (DT 19,4) con una media de 1,5 (DT $1,2)$ ingresos por paciente y presentaban una mortalidad del $6,6 \%$ durante el ingreso.

Cuando consideramos los pacientes que presentaban simultáneamente dos de las patologías señaladas, identificamos 10 grupos de pacientes que, en conjunto, sumaron 6121 sujetos (Tabla 2). En conjunto, tenían una edad media de 78,6 (DT 9,3) años, una media de 3,01 (DT 2,6) ingresos por paciente, la estancia total fue 251.224 días y la mortalidad intrahospitalaria acumulada 28.01\%. La asociación más frecuente fue EPOC e ICC que representó hasta el 2,6\% de la totalidad de los ingresos del área médica en el periodo señalado. Las mortalidades oscilaron entre $18 \%$ para el grupo con EPOC y Cl y el 39.4\% para la asociación EPOC y Cáncer.

Presentaron simultáneamente 3 de las 5 enfermedades seleccionadas un total de 1647 pacientes, repartidos en 10 subgrupos según las distintas asociaciones de enfermedades (Tabla 3). Su edad media global fue 80,1 (DT 7,3) años, 4,4 (DT 2,9) ingresos de media por paciente, estancia total 89.416 días y la mortalidad intrahospitalaria acumulada en el periodo señalado 35,68\%. La asociación más frecuente fue $\mathrm{Cl}$, ICC y EPOC, con un total de 459 pacientes $(0,9 \%$ del total de pacientes en el área médica)

Solo 265 pacientes presentaban 4 de estas 5 patologías (Tabla 4). En conjunto presentaban una edad media de 81.02 (DT 6,3) años, se registraron 5,5 (DT 3) ingresos de media por cada paciente con una estancia total de 17.417 días y una mortalidad acumulada del 39,2\%. Por subgrupos la asociación más frecuente fue Cl, ICC, EPOC y Cáncer (126 pacientes; $0,2 \%$ del total de pacientes). La mortalidad hospitalaria fue muy variable; desde el $17 \%$ para el subgrupo Cáncer, Demencia, Cl y EPOC hasta del 52,2\% para el subgrupo formado por los pacientes con Cáncer, Demencia, $\mathrm{Cl}$ y ICC. Por último, únicamente 18 pacientes presentaron las 5 enfermedades. Como grupo su edad media fue 80,3 (DT 6,2) años, 6,9 (DT 5,4) ingresos de promedio de ingresos, estancia total de 1.257 días y una mortalidad intrahospitalaria acumulada del $27,8 \%$.

\section{Discusión}

El 51,2 \% de los pacientes ingresados en el área médica de un hospital general presentan al menos una de las 5 patologías siguientes: Cardiopatía isquémica, demencia, ICC, EPOC o Cáncer. Las múltiples combinaciones entre ellas no sólo definen subgrupos de pacientes de alta complejidad cuyo manejo es todo un desafío clínico, sino también grupos de pacientes con múltiples reingresos, largas estancias y elevada mortalidad.

A pesar de las múltiples investigaciones que se han realizado sobre patologías crónicas concretas, existe muy poca información sobre los perfiles clínicos, las dificultades de manejo y la carga asistencial que suponen las diferentes combinaciones de estos procesos ${ }^{10}$. En 1995, en Estados Unidos, el $79 \%$ de las personas no institucionalizadas de 70 o más años sufría al menos una de las 7 condiciones crónicas más frecuentes: hipertensión arterial, enfermedad cardíaca, diabetes mellitus, enfermedad respiratoria, ictus, osteoartritis 0 cáncer ${ }^{11}$ y en hasta en el $20 \%$ de los casos coincidían dos o más de estos problemas. Sin embargo, entre los distintos

Tabla 2. Pacientes con asociación de dos enfermedades crónicas

\begin{tabular}{|c|c|c|c|c|c|c|c|c|}
\hline & \multirow{2}{*}{$\mathrm{N}^{0}$ de pacientes } & \multirow{2}{*}{$\%$ * } & \multicolumn{2}{|c|}{$\mathrm{N}^{0}$ de ingresos por paciente } & \multicolumn{2}{|c|}{ Edad } & \multirow{2}{*}{ Éxitus** } & \multirow{2}{*}{ Estancia total (días) } \\
\hline & & & media & DT & media & DT & & \\
\hline $\mathrm{EPOC}+\mathrm{CA}$ & 1.098 & 2,1 & 3,2 & 3 & 73,8 & 9,8 & $39,4 \%$ & 37.763 \\
\hline ICC + EPOC & 1.355 & 2,6 & 3,9 & 3,4 & 78,1 & 9,8 & $34,2 \%$ & 64.905 \\
\hline $\mathrm{Cl}+\mathrm{ICC}$ & 955 & 1,8 & 3,5 & 3 & 77,3 & 10,4 & $26,8 \%$ & 37.183 \\
\hline $\mathrm{ICC}+\mathrm{CA}$ & 565 & 1,1 & 3 & 2,7 & 77,6 & 10,7 & $38,6 \%$ & 20.141 \\
\hline $\mathrm{Cl}+\mathrm{EPOC}$ & 545 & 1 & 3,2 & 3,1 & 74,8 & 10,1 & $18,0 \%$ & 18.989 \\
\hline $\mathrm{ICC}+\mathrm{DE}$ & 526 & 1 & 2,8 & 2,0 & 84,8 & 6,7 & $37,5 \%$ & 18.768 \\
\hline $\mathrm{DE}+\mathrm{EPOC}$ & 506 & 1 & 3,1 & 2,8 & 82,2 & 7,1 & $30,6 \%$ & 19.162 \\
\hline $\mathrm{Cl}+\mathrm{CA}$ & 464 & 0,9 & 2,9 & 2,3 & 74,1 & 9,8 & $22,4 \%$ & 13.503 \\
\hline $\mathrm{DE}+\mathrm{CA}$ & 401 & 0,8 & 2,4 & 1,8 & 82,4 & 7,4 & $35,7 \%$ & 12.784 \\
\hline \multirow[t]{2}{*}{$\mathrm{Cl}+\mathrm{DE}$} & 232 & 0,4 & 2,7 & 2,7 & 81,5 & 7,3 & $23,7 \%$ & 8.026 \\
\hline & 6.121 & & & & & & $28 \%$ & \\
\hline
\end{tabular}

* \% de la totalidad de los ingresos. ${ }^{* *}$ acumulado en el total de ingresos

Cl: Cardiopatía Isquémica. ICC: Insuficiencia Cardíaca. DE: Demencia. EPOC: Enfermedad Pulmonar Obstructiva Crónica. CA: Cáncer de cualquier localización 
Tabla 3. Pacientes con asociación de tres enfermedades crónicas

\begin{tabular}{|c|c|c|c|c|c|c|c|c|}
\hline & \multirow{2}{*}{$\mathrm{N}^{0}$ de pacientes } & \multirow{2}{*}{$\%^{*}$} & \multicolumn{2}{|c|}{$\mathrm{N}^{0}$ de ingresos por paciente } & \multicolumn{2}{|c|}{ Edad } & \multirow{2}{*}{ Éxitus } & \multirow{2}{*}{$\begin{array}{l}\text { Estancia total } \\
\text { (días) }\end{array}$} \\
\hline & & & media & DT & media & DT & & \\
\hline $\mathrm{Cl}+\mathrm{ICC}+\mathrm{EPOC}$ & 459 & 0,9 & 5,6 & 4,4 & 76,8 & 9,3 & $32,2 \%$ & 29.863 \\
\hline$I C C+E P O C+C A$ & 300 & 0,6 & 4,6 & 4,0 & 77,7 & 8,8 & $36,0 \%$ & 16.883 \\
\hline $\mathrm{Cl}+\mathrm{ICC}+\mathrm{CA}$ & 184 & 0,4 & 4,1 & 3,3 & 79,3 & 8,6 & $34,8 \%$ & 8.448 \\
\hline$I C C+D E+E P O C$ & 160 & 0,3 & 4,6 & 3,1 & 83,7 & 5,9 & $38,1 \%$ & 9.027 \\
\hline $\mathrm{Cl}+\mathrm{ICC}+\mathrm{DE}$ & 147 & 0,3 & 3,8 & 2,5 & 82,7 & 6,9 & $40,8 \%$ & 7.411 \\
\hline $\mathrm{Cl}+\mathrm{DE}+\mathrm{CA}$ & 129 & 0,2 & 4,2 & 3,8 & 75,8 & 10,4 & $37,2 \%$ & 5.903 \\
\hline $\mathrm{DE}+\mathrm{EPOC}+\mathrm{CA}$ & 94 & 0,2 & 3,4 & 2,3 & 81,6 & 6,1 & $40,4 \%$ & 4.566 \\
\hline $\mathrm{ICC}+\mathrm{DE}+\mathrm{CA}$ & 89 & 0,2 & 3,2 & 2,2 & 83,9 & 6,3 & $36,0 \%$ & 3.841 \\
\hline $\mathrm{Cl}+\mathrm{DE}+\mathrm{EPOC}$ & 52 & 0,1 & 3,6 & 2,2 & 80,3 & 8,1 & $30,8 \%$ & 2.351 \\
\hline \multirow[t]{2}{*}{$\mathrm{Cl}+\mathrm{DE}+\mathrm{CA}$} & 33 & 0,1 & 2,8 & 1,4 & 80 & 6,1 & $33,3 \%$ & 1.123 \\
\hline & 1.647 & & & & & & $35,6 \%$ & \\
\hline
\end{tabular}

*\% de la totalidad de los ingresos

Cl: Cardiopatía Isquémica. ICC: Insuficiencia Cardíaca. DE: Demencia. EPOC: Enfermedad Pulmonar Obstructiva Crónica. CA: Cáncer de cualquier localización

análisis poblacionales efectuados se han apreciado notables diferencias. Marengoni'2 , en un estudio sobre 33 poblaciones seguidas durante 18 años, apreció que la prevalencia de MM entre los mayores de 65 años oscilaba entre el $21 \%$ y el $98 \%$. Otro estudio ${ }^{13}$, de similares características, encontró tasas oscilantes entre el $50 \%$ y el 100\%. En ambos casos estas variaciones son debidas no sólo a las diferencias entre las distintas poblaciones estudiadas, sino también a las diferencias en la obtención de los datos, los grupos de edad investigados 0 las definiciones usadas de MM. Entre los pacientes ingresados en un hospital, se estima que la media de problemas médicos por paciente es de 5 y que sólo un 15\% tienen un único problema médico bien definido. En estos pacientes, la edad y la presencia de comorbilidades se asocian con mayor necesidad de cuidados, mayor número de reingresos y, también, mayor mortalidad ${ }^{14}$. Además de la edad, la presencia de MM se incrementa en el contexto de determinadas patologías. Por ejemplo, el 49\% de los pacientes con ICC tienen 3 o más patologías concomitantes ${ }^{15}$.

A pesar de ser un problema muy frecuente y de gran repercusión sobre los servicios sanitarios, también es un fenómeno poco estudiado. Si bien existen numerosos estudios de pre- valencia, la ausencia de consenso no sólo sobre cómo definir, sino también sobre las formas de medir estos conceptos, limita la interpretación y la comparabilidad de los resultados ${ }^{16}$. Por ello, ante la ausencia de un criterio claro, en nuestro estudio decidimos analizar el peso de un número limitado de patologías que, desde un punto de vista clínico y a juicio de los investigadores, eran muy relevantes. La selección final se basó no sólo en la frecuencia, ya que todas ellas afectan a entre el $10 \%$ y el $20 \%$ de los pacientes en nuestra serie. Otras razones fueron: que son, por sí mismas, causas de ingreso hospitalario, que pueden ingresar en diferentes servicios del área médica por diferentes motivos, que generan una enorme complejidad asistencial y que todas disponen de guías de práctica clínica.

Por otra parte, los estudios prospectivos y bien diseñados sobre el impacto de la MM en los servicios de salud son muy escasos. Gijsen et al ${ }^{17}$, en una recopilación de 82 estudios de muy diferente naturaleza, señalaron que los pacientes con MM tienen mayor mortalidad, discapacidad y peor calidad de vida. También se apreció el incremento de uso de los servicios sanitarios (número de vistas al médico, hospitalizaciones y duración de la estancia) ${ }^{17}$ y mayor polifarmacia y reaccio-

Tabla 4. Pacientes con asociación de cuatro enfermedades crónicas

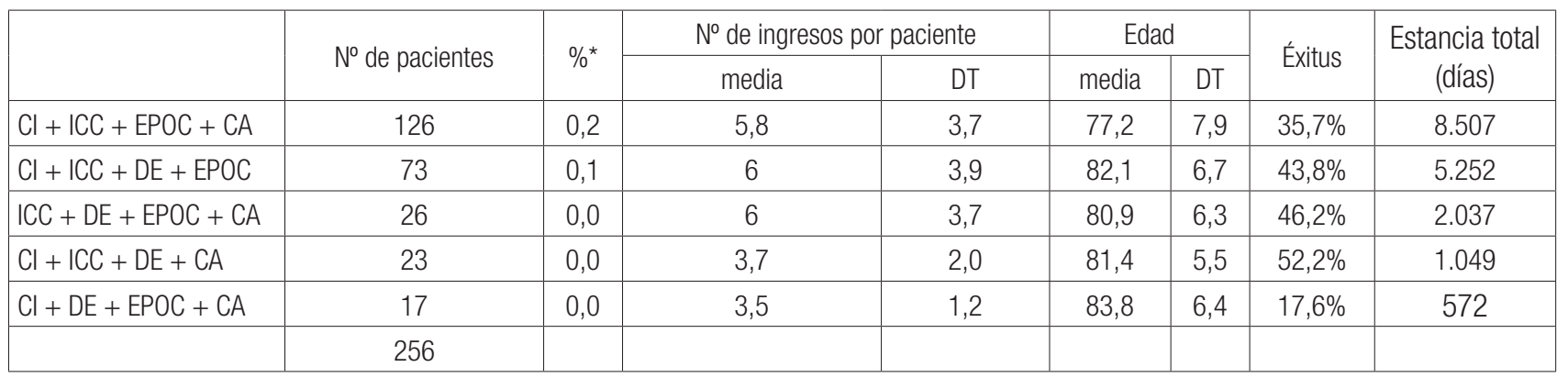


nes adversas a fármacos ${ }^{18}$. Nuestro estudio aporta que los pacientes con MM suponen un elevado porcentaje de las hospitalizaciones del área médica, independientemente de servicio donde se ubican. Por ejemplo, sólo las estancias de los pacientes con 3 patologías concomitantes (ver Tabla 3) equivalen, en un Centro como el nuestro, a la ocupación de 24 camas, todos los días del año, durante 10 años. Evidentemente los costes de esta asistencia son muy elevados. Sin embargo, más allá del precio, existen otros problemas, clínicos y humanos, más importantes. En primer lugar, ¿tenemos la estructura asistencial y el conocimiento clínico adecuados para manejar esta complejidad?. En segundo lugar, buscar nuevas fórmulas asistenciales que permitan mejorar la calidad de vida de los pacientes y, como consecuencia, reducir sus reingresos. En este contexto han surgido grandes iniciativas, como Partnerships for Patientes, programa federal en U.S.A., cuyo principal objetivo es reducir las hospitalizaciones evitables en un 20\% antes de $2014^{19}$. En nuestro país, y a nivel clínico, los programas de atención a patologías concretas, como los puestos en marcha bajo el auspicio de la Sociedad Española de Medicina Interna en la ICC, han demostrado mejorar la situación de los pacientes y reducir los reingresos ${ }^{4,20}$.

Los resultados de nuestro estudio han de analizarse teniendo en cuenta posibles limitaciones. En primer lugar, dado que cada Centro tiene su propia dinámica de trabajo, sus resultados podrían no ser extrapolables a otros Centros. Si bien los sistemas de trabajo pudieran ser muy distintos en Centros de muy diferente tamaño, en los similares el grado de estandarización de cuidados es muy alto por lo que los resultados no debieran de ser muy diferentes. Tampoco la población a la que prestamos asistencia y su distribución de enfermedades muestra diferencias sustanciales con las de su entorno ${ }^{21}$. En otro orden de cosas, puesto que los datos se recogen de las bases de datos del hospital pudiera pensarse que existan errores de clasificación. Sin embargo es altamente improbable; la inclusión de los diferentes procesos en las bases de datos se hace utilizando el informe de alta como fuente y por profesionales con experiencia, con una cualificación similar a los de su entorno y utilizando procedimientos normalizados de trabajo muy estandarizados. Por todo ello entendemos que si bien pudieran existir diferencias con otros Centros de nuestro entorno, estas han de ser poco relevantes y que los resultados generales y tendencias de estudios similares en otros Centros debieran ser superponibles a los nuestros.

En resumen, los pacientes con MM, en sus múltiples combinaciones y variantes, suponen un porcentaje muy elevado del trabajo asistencial del área médica de un Hospital, con múltiples reingresos y elevada mortalidad. Todo ello supone no sólo un desafío clínico en el manejo de patologías muy diversas y complejas, sino también un importante problema de gestión. Nuestro estudio identifica subgrupos de pacientes que suponen un porcentaje muy elevado de la actividad hospitalaria y donde es posible establecer programas que mejoren su calidad de vida y reduzcan sus ingresos hospitalarios.

Estudio financiado con una Beca de investigación de la Sociedad Gallega de Medicina Interna. Convocatoria 2010.

\section{Bibliografía}

1. Akker M van den, Buntinx F, Metsemakers JF, Roos S, Knottnerus JA: Multimorbidity in general practice: prevalence, incidence, and determinants of co-occurring chronic and recurrent diseases. J Clin Epidemiol 1998, 51: 367-75.

2. Fortin M, Lapointe L, Hudon C, Vanasse A, Ntetu AL, Maltais D: Multimorbidity and quality of life primary care: a systematic review. Health and Quality of Life Outcome, 2004; $2: 51$

3. Zapatero A, Barba R, por el Grupo de Gestión Clínica FEMl. Estudio de un millón de altas hospitalarias en Medicina Interna. Disponible en www.fesemi.org/grupos/gestion clinica/noticias/un millon de altas.pdf

4. Cerqueiro González JM, Terrón Pérez F, Abeledo Vázquez C, López Díaz MJ, Matesanz Fernández M, Casariego Vales E. Resultados asistenciales de una consulta monográfica para pacientes con Insuficiencia Cardíaca. Estudio de Cohortes. Galicia Clin 2010; 71: 103-110

5. Nagel G, Peter R, Braig S, Hermann S, Rohrmann S, Linseisen J. The impact of education on risk factors and the occurrence of multimorbility in the EPIC-Heidelberg cohort. BMC Public Health 2008, 8: 384; doi: 10.1186/1471-2458-8-384.

6. Bengoa R. En Curar y cuidar. Innovación en la gestión de enfermedades crónicas: una guía práctica para avanzar. Bengoa R y Nuño R eds. Elsevier Masson, Barcelona 2008.

7. Charlson ME, Pompei P,Ales KL, MacKenzie CR. A new method of classifying prognostic comorbidity in longitudinal studies: Development and validation. J Chronic Dis 1987; 40: 373-383.

8. Hudon C, Fortin M, Vanasse A. Cumullative IIIness Rating Scale was a reliable and valid index in a family practice context. J Clin Epidemiol. 2005; 58: 603-608.

9. Schäfer I, Hansen H, Schön G, Maier W, Höfels S, Altiner A et al. The German MultiCare-study: Patterns of multimorbidity in primary health care - protocol of a prospective cohort study. BMC Health Serv Res. 2009 11; 9: 145. Disponible en: http://www. biomedcentral.com/1472-6963/9/145.

10. Vogeli C, Shields AE, Lee TA, Gibson TB, Marder WD, Weiss KB et al. Multiples chronic conditions: prevalence, health consequences and implications for quality, care management and costs.. J Intern Med 22 (Suppl 3): 391-395.

11. Centers for Disease Control and Prevention. Unrealized Prevention Opportunities: Reducing the Health and Economic Burden of Chronic Diseases. Atlanta, GA; Centers for Diseases Control and Prevention; 1997.

12. Marengoni A: Prevalence and impact of chronic diseases and multimorbidity in the aging population: A clinical and epidemiologicalapproach. Stockholm: Karolinska Institutet; 2008.

13. Mercer SW, Smith SM, Wyke S, O'Dowd T, Watt GCM. Multimorbidity in primary care: developing the research agenda. Fam Pract 2009; 26: 79-80.

14. Bernabeu M, Ollero M, Moreno L, Barón B, Fuertes A, Murcia J et al. Development of a new predictive model for polypathological patients. The PROFUND index. Eur $J$ Intern Med 2011; 22: 311-317.

15. Abeledo C, Gómez E, Terrón F, Cerqueiro JM, Casariego E. La consulta de enfermería en el control de pacientes con insuficiencia cardíaca. Libro de Comunicaciones del II Congreso Nacional de Atención al Paciente Crónico, Santiago de Compostela, 2010: 29.

16. Valderas J, Starfield B, Sibbald B, Salisbury C, Roland M. Defining comorbidity: Implications for understanding health and health services. Ann Fam Med 2009; 7: 357-363.

17. Gijsen R, Hoeymans N, Schellevis FG, Ruwaard D, Satariano WA, Bos GA van den: Causes and consequences of comorbidity: A review. J ClinEpidemiol 2001, 54:66174.

18. Budnitz DS, Lovegrove MC, Shehab N, Richards CL. Emergency hospitalizations for adverse drug events in older americans. N Eng J Med 2011; 365: 2002-12.

19. HealthCare.gov. Partnership for patients: better care, lowest costs. Washington, DC: Department of Health and Human Services (http://www.healthcare.gov/center/programs/partnership/index.html)

20. Anguita M. Programas de intervención en la insuficiencia cardiaca: análisis crítico. Rev Esp Cardiol 2007; 7: 45 - 56

21. Servicio Galego de Saúde. Memoria 2009 Servicio Público de Saúde de Galicia. Santiago de Compostela, 2011. Disponible en: http://www.sergas.es/Publicaciones/ DetallePublicacion .aspx?ldPaxina $=40008 \&$ IDCatalogo $=2053$. 